\title{
Structure and communications in the process of organisational change: Eastern European experience and its general relevance
}

\author{
P.C. Humphreys
}

London School of Economics \& Political Science

Houghton Street, London WC2 2AE, UK

Tel +44171955 7711, Fax +441719557565

e-mail P.Humphreys@lse.ac.uk

\section{E. L. Nappelbaum}

Russian Institute for Systems Analysis

9 Pr 60 Let Octabryja, Moscow 117312, Russia

Tel/Fax +70952824275

e-mailernapp@aha.ru

\section{Abstract}

Every organisational change creates some organisational stress and makes personnel both winners and losers within the organization. To a large degree this is a result of the inevitable change of the organization perspective as well as ensuing organisational restructuring. In this paper we investigate the organisational perspectives generally adopted by top management, and explore the consequential stresses generated in the attempt to implement the ensuing prescriptions for change.

We stipulate that middle management will feel themselves the most threatened by processes of organisational change instigated from above. We demonstrate how they transfer this feeling further down the hierarchical ladder. A major factor here is a virtual break in communication within the organization resulting from the radical change of the communication context. We illustrate how situation is further aggravated by middle management assuming the role of interpreters of top management's intentions and of the sole guardian of the power of the organisational unit they manage and hence of the protector of its personnel. In this way the previous organisational structure becomes an infrastructure for the pockets of tacit resistance to organisational change that may frustrate its purposes in the long run.

On the basis of the model for problem formulation and choice developed by Nappelbaum, this situation is examined and is related to the issue of 
implementation design for a strategic decision taken by the upper management. It is stipulated that team building is an inherent part of this process and should involve not only those who instigate the change and prepare corresponding decisions but also every echelon of the organisational management.

This understanding imparts a new flavour to the notion of participative management and imposes new requirements on organisational communications and on computer support for organisational change. In this respect the concept of variable precision modelling of knowledge about local enactment processes (developed within SASOS by Humphreys and Berkeley) becomes especially relevant. Within this framework, however, the notion of zooming is to be rethought from aggregation/disaggregation to reflection and analytic reinterpretation and creative synthesis.

Theoretical ideas to be presented here are illustrated by the recent experience of attempts at organisational transformation and business development within the Hungarian reform process and Russian perestroika. We consider that the experiences reported here are quite relevant to organisations in general, wherever in the world they are located.

\section{Keywords}

Organisational change, decision conferencing,participative management, Eastern Europe, business practice.

\section{INTRODUCTION: THE CONTEXT OF ORGANISATIONAL TRANSITION IN EASTERN EUROPE}

Today, after nearly a decade from the beginning of the new era in Eastern Europe which started in the mid 1980's at the time of perestroika in Russia, one may justifiably regard the processes that are taking place there as (among many other and probably much more significant things) a huge and most comprehensive study in social and organizational change. True, many people, both from the right and the left of the political spectrum, claim that many of the changes are very shallow and superficial in their nature. This, however, is simply a debate over the depth of the changes implemented and over their lasting values and desirability. The scope of the changes and especially of attempts to instigate some change is clearly evident.

These processes of change - how a change was conceived, planned, prepared for, implemented, fought against, perceived, and evaluated - if deeply, carefully, and objectively studied, might offer an incredible wealth of material for both theoretical and practical analysis. However, this is rarely properly done or properly documented and for several very good reasons. The most important of those is political sensitivity of the very issues involved as any statement about a change at any level and especially assessment of its desirability, planned nature etc. would be immediately exploited by one political side and as swiftly challenged by an opposite one. But, from a methodological viewpoint, it is probably even more 
important that any "objective" assessment should inevitably involve a common "frame of reference". Most of the material that follows argues implicitly that any change is first and foremost is a change in the frame of reference and hence should be judged on its own merits.

Nonetheless several illuminating conclusions can be made even at the level of pure "unequipped" observations, especially if they are made at "embedded observation" basis. These conclusions may be especially emblematic as they cover a wide spectrum of change stakeholders and their attitude towards this change. Indeed, it is equally interesting to observe what changes top management of various enterprises see fit to introduce in response to the ever-changing business environment and how it goes about it or how people at the grassroots level adapt to the changes and assess them.

One of our more interesting observations is that rarely does implemented change result in the state of affairs that was the objective of the change. Moreover in many cases the result of a change is totally counterproductive with respect to the underlying intentions. It would be easy and tempting to explain this discrepancy by either a deficient planning of the change or by hypocrisy of top managers who proclaim one thing but perform something totally different. The reality, however, is in many cases much more complicated. Time and again it became obvious that managers planning to enact a change failed to understand that each major change involves also a drastic change of perspective of all the actors involved, of their frame of reference, as well as of an infrastructure stability of which quite often is a major precondition of the success of the change instigated. Moreover, an obvious complexity of the social texture where the change is being enacted very often results in appearance of "emergent" effects that are very difficult to attribute to tangible causes and even more difficult to forecast and to allow for.

Not surprisingly, given the above observations, the reform processes in most countries in Eastern Europe have been accompanied by a decline in industrial production. Initially, the underlying severity of this process was masked by a low level of apparent profitability. This was sustained on what were increasingly loss making operations by using up assets and resources. As such accumulated resources become exhausted, the pressure for organisational transformation and new business successes comes ever more to the fore as an essential survival strategy. Now this in evinced even in the pressure from some quarters for a "re-transformation" to earlier organisational forms, remembered with nostalgia after the failure of interim attempts at transformation.

This apparent nostalgia stems from the way in which people at large assess the results of a change even when they shared the underlying values in terms of which the change was considered desirable. If the enacted change was successful these values become a stable feature of the everyday environment, and in human consciousness transfer themselves to the realm of the behaviour infrastructure which is generally taken for granted and does not enter in an assessment pattern. The change induces a totally different frame of references, in which comparison 
with the previous state of affairs plays a crucial role. This state is often assessed in a highly idealised way by disregarding the features that were the most distressing and hence led to the change and by overestimating the features that cause distress now.

The interim attempts at transformation have been generally characterised by the decomposition of inherited, overcentralised organization structures. However the idea of organisational hierarchy, with rigid distinctions between top management, middle management and workforce has, at the same time, been reinforced. Mirroring the contemporary political struggles founded on a competition to the death between stereotyped political forms, the appropriate design for an organization has usually been perceived as an outcome of a struggle between various organisational forms, in which the management-workforce control hierarchy, with strict accountability between levels (Jaques, 1989), has generally assumed hegemony.

Some interesting observations can be made, in this respect, concerning the attitudes and behaviour of top management of various enterprises. The most evident feature of those is a very strong resistance to changes in their own enterprises even if they are dictated by the changed business environment. In principle, old guard longs for the changes that are purely cosmetic and increases only their own freedom of manoeuvre and their benefits leaving the rest of the business environment intact and predictable. Surprisingly, such an attitude finds an unexpected support in the inner dynamics of change itself. Indeed, such changes create transient business environments in which it is exceedingly difficult to orient and to operate. In this kind of environment, perhaps the only factor of lasting value is the previously established personal relationships that allows the directors of the emergent enterprise to recreate, to a large extent, many salient features of the old distribution system. This serves to promote and maintain the myth that the old "directors' club" are the only people capable of managing large-scale industrial enterprises. This, in turn, has led to the temporary revival of seemingly rejected political doctrines, as can be publicly witnessed at election time in most Eastern European countries.

This process has also been accompanied by decentralisation of production and services, and the formation of new kinds of alliances and ventures under the rubric of "privatisation". For example, we frequently find short-sighted behaviour on the part the management of those plants that outsource an enterprise that acts as a main breadwinner. They may raise the prices for the outsourced material they supply in an attempt to benefit inordinately from their firm's monopoly position: dealing with the main enterprise very much in the same way as they were previously dealing with the state. Such practices have brought leading enterprises to the brink of uncompetitiveness, potentially distorting the very source of their own financing.

By the same token, in forming their partnership relations with Western companies, Russian large-scale enterprises prefer to establish them with very largescale partners (sometimes clearly in disadvantage to their own good) which they try to deal with in much the same way they were accustomed to deal with the previous State owned enterprises. 
Quite a different style of business behaviour is demonstrated by younger people in setting up their new and generally small businesses, especially if they build from scratch and build them up gradually, employing (especially in the beginning) people who are fully aware of what they are employed to build. These small businesses are usually intended, by those who set them up, to thrive on the rapidly changing, transient business environment. When they are formed as joint ventures, the local directors characteristically find fault and express disappointment with the behaviour of their Western partners, whose minds are tuned to a much more stable and less opportunistic economic climate.

The entire organisational structure emerging as a result of these attempts, however, has proved typically to be highly unstable and prone to serious calamities. The resulting very high degree of failure (both explicit and latent) among the newly created organisations is a good indicator that there is much which is fundamentally wrong with the current attitude to organisational transformation amongst managers operating in Eastern Europe, and probably elsewhere too.

\subsection{The negative inheritance}

To some extent, the roots of these failures can be traced to a heavy negative inheritance from past systems and in the lack of much attempt to understanding this inheritance (rather than trying to wipe it out of memory) and of its influences on the organisational future. Key issues from the past with negative carry-over to the present are:

- Within the "first economy" there were generally negative incentives for good, innovative management: Failures were attributed personally but success were attributed collectively; project management was limited to technical development rather than to managing all aspects of innovative projects, viewed as socio-technical and human activity systems. Risk analysis techniques were highly developed but only on an informal, not on a formal basis. Moreover the focus was on the analysis of political risk rather than business risk.

- Within the "second economy" there were, of course, economic rewards to be gained form the personal exercise of initiative and innovation, but such efforts were generally limited to commerce and were often clandestine. Within this sector, the mechanisms of production, where they were existed, were highly distorted and were generally parasitic on operations located within the first economy. Associated with the clandestine nature of these activities was the importance of private knowledge. Knowledge about "how to" was a commodity best kept in shortage, rather than to be shared publicly.

In general, very little pre-competitive infrastructure existed within the second economies flourishing in Eastern Europe, and that which existed within the first economy died with it. Its death throes were, for the reasons outlined above, 
accompanied by the subversion for the basis of trust within any organisational forms which would newly emerge, whatever their nature.

\subsection{Importation of Western business practice ideas}

The reasons for failures in organisational transformation and business development should not, however, be sought solely in the past. No less important reasons can be identified in shortcomings of the new ways of doing things in organisations, and of doing business in Eastern Europe. Prescriptions and the rationale of originating in Western business practice were, and continue to be, imported completely out of context. Scant attention is given to the differences in infrastructure on which business, private and even political life is based in the particular national context, compared with the context in which the imported prescriptions originated.

The pressure and the agency for this process of importation and reimplementation of perceived successful "Western" business techniques lies, increasingly, with those personnel who assume the "top management" of the emergent organisational units, even if they did not occupy such positions before. These are, typically, people having the agency and financial backing to perform "management buy-outs"; to liaise with venture capitalists and interpret their wishes; to form "joint ventures" with western partners, and so on. It is our experience (supported by Paprika and Zoltay, 1994) that, when help is sought by such people for professional experts, analysts and consultants in decision and management support, their first priorities are for support in regard of:

- Innovative marketing solutions, rather than market research;

- Brainstorming on new ventures;

- Negotiations with potential partners, banks, etc.;

- Techniques for increasing workforce efficiency while maintaining morale within a climate off staff cut backs.

The conjunction of these four priorities stems from a general belief that because the organization has, in the past, been successful in operational terms (provision of a specific type of product, service, etc.), the aim must now be to maintain and even enhance the success of the old, continuing, "operational core" in the newly emerging economic, social and political context.

What is not addressed in this view is the need for an understanding of why the organization was successful in the past, not just in operational terms, but considering all the kinds of resources in its constituent human activity system. Only then is it possible to understand how the resources currently surviving in the organization can, collectively, form the basis for its future success. Such an understanding involves a completely different conception of what is the "core" of an organization. This is something which we will explore further in section 3. 


\subsection{The split between organization and workforce}

The priorities discussed in the previous section also reflect the concerns of top management within a perspective on their business which enshrines a fundamental split between organisational structures, which appear as autonomous with a life of their own, and human practices within organisations which appear as apart: thrown off centre from the decision making process. This perspective also promotes management-centrism: Management makes decisions; Management transforms the organization (Merkle, 1980). Attempts may be made to use participative techniques like Kaizen (Masaaki, 1989) among groups of workers and front line managers to boost morale, within a perspective which dissociates subjectivity frorn organisational tasks. Thus it seems to be a surprise to top management when such activities fall apart, in terms of their stated aims, when their own view that "the bottom line is to reduce staff costs" becomes common knowledge amongst the participants.

This is not to say that we are witnessing a return to "scientific management" according to the theories of F. W. Taylor (c.f. Taylor, 1947; Nelson, 1980). In the post-modern world, Taylorism is discredited in both Eastern and Western European management practices, being blamed for the development of distorted, fragmented and inhumane work methods of work practice during the period of modernist excesses in productive enterprises in both Western Europe (Morgan, 1986) and Eastern Europe (Beissinger, 1988), as well as in the United States (Wrege and Greenwood, 1991). Currently, in both West and East Europe, the impetus is on the replacement of the remains of Taylorism with the "Human Relations" approach which seeks to:

" reshape the internal world of the organization so as to release the autonomous subjectivity of the worker in such a way that is aligned with the aspirations of the enterprise, now construed in terms of innovation, flexibility and competitiveness" (Rose, 1989).

We hear from top management, everywhere in Eastern Europe, a growing tendency to insist that development and transformation of their organisations should be made in the service of "increasing innovation and competitiveness," but the divorce of their workforce from the concept of their "organization" still remains in the discourse and practice of this level of management. Thus subjectivity is dissociated from organisational settings. The result is that the managers and their organisational analysts are located in professional roles, external to, and alienated from, the organisational process itself (Humphreys, Berkeley and Jovchelovitch, 1996). 


\section{TOP MANAGEMENT'S DESIRES FOR DECISION SUPPORT}

A decade ago (Humphreys, 1984) we identified an almost complete absence in practical applications at top management level of interactive computer-based systems based on traditional DSS and IS design methodologies (Lederer and Sethi, 1988), a situation which continues today. We stated then that

"the problem here is not simply a failure of decision support systems at higher levels. It may not be wise to attempt to formalise scenario generation techniques and problem structuring languages into automated decision support systems....Decision makers' scenarios need to be explored rather than be fitted into formal structures...It is better to develop techniques for the psychological validation of the decision makers' own problem structuring language than to try to invent a universal problem structuring language that will have to be taught from scratch to high level decision makers." (Humphreys, 1984, p. 20)

Phillips (1989) identified two approaches to GDSS for top management which were then beginning to emerge as a. result of the failure of the traditional DSS/IS approach. The first followed from the view that "the most fundamental activity of group decision making is interpersonal communication and the primary purpose of a GDSS is to improve group communication activities" (DeSanctis and Dickson 1987), and sought to provide a computer-based workbench environment with the intention of facilitating group communication. Phillips claimed that this approach was inferior to the second approach which "provides a problem solving environment that is group centred and is primarily intended to help managers consider uncertainty, form preferences, make judgements and take decisions".

The latter approach, usually implemented at top management level in the form of "decision conferencing" (Phillips, 1992), has increasingly become the preferred approach to import from the West in the service of East European top management's desire for support in their efforts at organisational transformation (Fekete-Szucs, 1991; Vari, Rohrbaugh and Baaklini, 1992). Part of the reason for this preference is the recognition the particular validity in the context of the problems facing Eastern Europe of Phillips' (1989) claim that "concentrating only on the technology, computers, software and networks will not reveal the full potential of GDSS".

Phillips' criticism has generally been taken as a reason to dismiss the first approach on a wholesale basis but, in our opinion, this is unfair. The criticism only applies to a particular implementation strategy which is more appropriate in the cultural and economic context of Texas than of Hungary or Russia, and we will return, later in this paper, to the fundamental importance of improving group communication activities. First, though, we examine in more details the consequences of the hegemony of top management, following the second, approach for implementation of organisational transformation. 


\subsection{Consequences of planning organisational transformation through top management decision conferencing.}

In decision conferences focused on organisational transformation, the participating top managers usually first plan the restructuring of the organization, reaching agreement on goals, strategies and future scenarios within which the chosen strategy appears to unfold successfully, and then calculate staff losses, redeployments, etc. (Bannon, 1997) Finally, agreement is reached on action. This usually means allocating and confirming responsibilities among the individuals present on the prescriptions each should issue to his or her subordinate managers in order to initiate the implementation process which, it is believed, will secure the desired changes (Fekete-Szucs, 1991; Phillips, 1992). The implementation process itself is usually not considered in any detail in the conference, as the necessary expertise is located at lower levels within the organisational process, split off from the experience and management concerns of the participants in the decision conference.

Our experience is that this agenda for decision conferencing tends to have the following unfortunate concomitants:

- Failure within the implementation scenarios to identify properly and provide for handling side effects of the main, intended, effect;

- "Throwing the baby out with the bath water" through focusing on the rolling back of initial scenarios without the opportunity to create innovative pathways to goals, as they lie off the roll-back route;

- Missing of opportunities and creation of problems for change implementation management;

- Underestimation of the value of (or even the existence of) local skilled knowledge in place within the organization.

These drawbacks are omnipresent not only in formal decision conferencing but also in the very formal and informal activities that enact a change and hence are very central in understanding some failures to implement a change and in improving the efficiency of the change management in general. They are as well as many other drawbacks are intimately related to a "linear mode of thinking about change" that remain prevailing in our theory and practice and in the final analysis of a linear model of choice and problem solving limitations of which were exposed in an old but still quite topical paper that is not very well known and which is published for the first time in this volume (Nappelbaum, 1997).

The main flaw of the linear way of thinking about change lays in its "measurement paradigm", a contention that the problem to be solved to enact a change and hence a change itself is some how "given", objectively present in the 
existing problem environment (or "problematique" to use a term popular in systems research) and that the problem of the management is to recognise it and to find the efficient way of its implementation.

This model of thinking does not recognise that the problem environment itself is nothing but a very subjective and very limited perception of a much more complex and much less manageable environment. Moreover this perception is not a mere partial mapping of this reality into a manageable representation but a result of a purposeful creative design effort involving complicated manipulation of the available data and establishment of powerful restrictions one of the major objectives of which is to make the representation manageable.

Nothing in this representation is "given": nor the way in which we describe the states we want to change as well as the state space boundaries, nor the frame of reference to be used in assessing whether a change is desirable or not, nor the scope and the content and efficiency of actions we are willing to allow in order to enact a change.

The mere fact that this representation exists and looks "natural" and unique is a good indication of its stability and previous success and there is a good chance that the desire for change is a manifestation of such a turn of the affairs that made this same representation suspect and not nearly as successful as it was in the past. Obviously any attempts to find the remedy within the framework of such a representation is doomed to failure and may eventually jeopardise the very idea of a change. It is in this sense we have alluded several times above that a real change (rather than a mere dynamics) involves always and foremost a change of the frame of references and even of a perception of the "objective" reality.

Another reason why the original representation appears to be an "obvious" one and a unique one is its holistic, robust nature. In (Nappelbaum, 1997) this is explained by an inner balance between its various components achieved during the original design of this representations, by its "cognitive closeness" requiring people who share this perception to reinforce in their thinking and their behaviour the validity of this perception very much in the same way in which "organizational closure", as described by Maturana and Varela (1979) is preserved. It is this inner holism and closeness of a perception that is responsible for a well-known effect of incomparability and irreconcilability of different personal attitudes with which every decision conferencing has to deal at some time.

Recognising a positive value of the difference in personal perceptions and at least on the face of it paying a special attention to a deviate opinion current conferencing techniques deal with the plurality of personal perceptions in a most negligent and cavalier manner. The only way in which the existing methodology knows how to enrich a perception consists of a mechanical merger of the attributes belonging to different cognitive closures and in looking for trade-offs between different assessments. In doings so, however, we destroy underlying tightly knitted structures of the merged perceptions and replace them with their flattened and hence placid projections, losing on the way some extremely rich semantic information. 
Moreover, expanded perception cannot be balanced by definition and hence a superficial representation that we are trying to use for the search of a viable strategy lacks the very vitality, integrity and persuasive power so much needed if we want the designed strategy of change to be successful. We have argued (Humphreys and Nappelbaum, 1989, Nappelbaum, 1997) that a genuine approach to expansion of the perception of a problem environment can be only achieved through designing for the same objective reality as many competing and mutually incomparable representations as possible and to transfer a choice between various options to the choice between various perceptions each of which suggest an obvious solution of a problem it is expressing.

- In other words, a problem can be considered as "properly formulated" only if we know (or believe, probably erroneously) how to solve it and what is this solution.

- The issue, however, is to chose or more precisely to design, a problem we believe we need to solve.

- This choice, in its own turn, require involving perception of a higher level which may call for the rethinking of the very principles that the enterprise is built upon.

Thus, if we are to avoid the third and fourth "unfortunate concomitants of decision conferencing", described above (i.e., missing of opportunities and creation of problems for change implementation management; underestimation of the value of local skilled knowledge in place within the organization), this calls, first of all, for a profound analysis of the existing system of values constituting the very interorganizational culture and checking its consistency with the value systems attached to every particular solution and its underlying perception.

This is a very important and an often neglected feature of organization of knowledge needed for planning and implementing a change. Each action which is going to be taken to implement a change, however small, brings with itself its own "native" perception involving its own frame of reference, its own system of values, its own set of instrumental intentions etc. And all this components belonging to perceptions of different levels should be innerly consistent. Their intrinsic inconsistency may result in emergent side effects that in the final analysis may jeopardise the very intentions for which the change was enacted.

That is why a detailed implementation plan - the implementation definition cycle - to add to the original problem definition and situation definition cycles should be an indispensable part of any change management scenario. Such a cycle should not only to check whether we are dealing with a case of a "collective fantasy" (Humphreys and Berkeley, 1986, Humphreys, 1989) or the suggested solution is actually implementable but also to check the price of this implementation and not only in terms of the resources allocated but also in terms of the values compromised. 
Consideration of the implementation definition cycle reveals also yet another extremely important dimension of the change management - an issue of stakeholders (Mitroff, 1983). Usually decision conferencing takes notice of the personal differences of a very limited circle of stakeholders - comprising mostly those who plan and manage the change process. In organizational setting, however, a real set of stakeholders are much larger and encompass both the actors and the "consumers" of the change and each of them brings into the process its own perception of that part of the overall reality that is relevant to them. Inconsistency of the "organizational" and individual perceptions and especially of its value components may destroy the manageability of the change process and compromise the entire endeavour.

Implementability of any change process and even its relevance is a very provisional thing strongly dependent on the existence and stability of the underlying infrastructure. In terms of the circular logic of choice (Nappelbaum, 1997) this infrastructure can be defined as those features of perception that are taken for granted and whose stability should not be affected by the planned change. It is precisely because of this uncontested nature of our beliefs about the existence and stability of the relevant infrastructure that makes its analysis so difficult and so rare. Design and analysis of alternative perceptions besides one that is used for the purpose of change management and planning can be instrumental in finding what is assumed to already exist or to happen by itself, what are the foundation for such believes and what external factor may affect the validity of such assumptions. In section In section 3, we will discuss how this may be done, taking into account the knowledge and subjectivities of all the participants involved in the transformation process.

\subsection{Neglect by top management of situation and implementation definition cycles}

We have shown above how unfortunate concomitants of decision conferencing follow from the usual practice of considering only the problem definition cycle within the three cycle scheme identified by Nappelbaum (1997) as essential for effective utilisation of the full range of relevant information and expertise existing in the organization in the planning, managing and implementation of the organisational transformation process. The neglect of the other two cycles in the scheme stems from the hegemony of top management's desires for change at the core of the planning for organisational transformation.

The situation definition cycle is neglected because handling information about the organization within this cycle means keeping aware of the recent history of processes and participants at all levels in the organization: something which senior management usually (and incorrectly) seek to identify as the core of the negative inheritance which is responsible for their current dis-ease.

The implementation project definition cycle is neglected as a consequence of splitting off the notion of the organization and its transformation from the 
subjectivity of those members of the organization who actually implement the transformation through the collective changes in their communications and work practice. Hence implementation project managers and their teams perceive the outputs of top management's decision making as requirements for changes and deliverables: They must analyse these requirements and implement the processes which will result in the deliverables within constraints set from above (Berkeley, De Hoog and Humphreys, 1990), but the possibility of generation of the requirements and possibilities for transformation is excluded at the levels in the organization where it should actually occur, rather than just be planned and prescribed.

In the following section, we examine the consequences of this neglect, focusing particularly on how it can destroy the participation of middle management in developing any kind of effective organic transformation process.

\subsection{The problems of middle management}

It is well known that adoption of a new policy generally entails structural changes; also the existing structure significantly constrains the type of strategy that an organization is willing and capable of adopting (Chandler, 1969). The latter is most heavily related to a mismatch between the top-level management of the organisational perspective and lower-level ones that may emerge at the stage of development and implementation of a new organisational strategy. We will refer to that issue as to the problem of vertical or structural implementability. The need to consider this implementability dimension stems from the fact that organization stakeholders and actors are by no means obliged to take new organisational objectives and policies, prescribed from above, in their stride. Rather, they tend to consider these as annoying constraints in their efforts to keep performing those activities which they know best how to perform and to change only the appearance rather than the gist of the change as conceived from above.

The vertical implementability issue can be further aggravated by structural stability which is built into every viable organization (Espejo, 1989). In principle, a change of the policy that inevitably involves a change of perspective and implies a change of the organization structure, unavoidably leaves some of the members of that organization worse off. More or less by definition, the most unfavourably affected and endangered strata of these members are middle management (Keen, 1986).

Indeed, top managers in an organization undergoing a change of structure, following the "preferred approach to decision making we identified in section 2.1, are likely to be more flexible in their capabilities, and better prepared for the changes which are planned to take place as they tend to be the owners of the transition problem. Similarly, the lower "technological" levels of the organization tend to be the "physical" incarnation of the organization competence. Hence the physical, technical, capabilities existing at these levels will be formulated in the 
course of the new policy development and, thus in principle, should be allowed for in the planned organisational transition.

Middle management, however, tends to represent the organization structure itself. Changes in organization strategies and the ensuing modifications in the organization structure inevitably imply redistribution of organisational power and, even more seriously, the functionality of various elements of organisational structure, to which middle management may be unwilling or even unable to find an easy answer, or any answer at all.

This situation is further aggravated by the fact that middle management traditionally constitutes the main network through which decision made by top management are transferred to the lower levels of the organization for implementation (Jaques, 1976). In making this transfer, middle managers do not merely pass the information along but also translate from the "strategic" (top management) language in which it is received into prescriptions and instructions in a language which they think will be understood by the "technical level" recipients of their communications lower down the organization (Jaques, 1989). In doing so, they must interpret this information (Cooper and Burrell, 1988; Checkland, 1994) and, through this traditional role middle managers obtain the possibility of distorting this information in the light that is beneficial to themselves.

In the cases of organisational transition we have studied in Hungary and Russia (and elsewhere, for that matter), top management usually turned out to be much more interested in finding an appropriate policy, and an appropriate set of objectives, and perspectives, rather than in team building and in propagation of their new vision through all the levels, right to the bottom of the organisational ladder. A perspective developed at the top level is generally very difficult to translate into the context that rules the behaviour of those who operate at much lower levels in the organization. They need time and effort in order to be able to develop an outlook that is well adjusted to the new top-level perspective (Yetton and Crawford, 1992). Moreover the amount of time and effort required to do this at the lower levels is usually much greater than that which was needed at the top level. The translation and implementation process is especially difficult at lower levels due to the lack of specialists in the highly creative business of planning organisational transformation. Most people employed at the technical level are much more accustomed to think in stereotypes which have been enforced by their entire previous life in the organization, and which are now being negated by the organization transition under way.

In such an environment of uncertainty and ensuing anxiety middle management get the chance to rally the support of the lower, largely disaffected strata in their organization in their efforts to resist those structural changes planned by top management that may remove them from the power or dilute their power. In calling for protection of the existing structural entities they lead, they portray their activity as a natural defence of previous "traditional" values as well as of the vested interests of the group they represent. In this way they hamper the very process of 
adaptation of the lower levels of organization to the new ideas born at the upper levels.

\section{STAKEHOLDERS IN THE LIFEWORLD OF THE ORGANIZATION IN TRANSFORMATION}

Considerations of the sort discussed above give a totally different meaning to the idea of participative management (Mumford, 1991). Now it becomes evident that, even at the stage of the development of the new policy, focusing on DSS to support the concerns and desires of top management is insufficient. One has to allow for the entire plethora of the new, more focused, perceptions and local policies that would inevitably emerge in the course of new policy implementation. These somehow have to be consistent with the "grand plan" of the organisational transition, conceived on the basis of larger strategic considerations applicable to the organization as a whole. It is no longer enough just to invite some representatives of the lower levels of management and of the workforce to take part in the development of this grand plan.

It is equally essential to give time and opportunity to all the stakeholders and actors to develop their own adjusted perceptions and policies and for checking the internal consistency of these perceptions and plans with the aggregated (rather than prescribed) one. These stakeholders and actors may occupy not only the roles of problem owner, decision maker, expert, i.e., those familiar to the participants in decision conferences on organisational change (Vari and Vecsenyi, 1984), but also a host of other roles in the organization lifeworld, reflecting the fact that they are, in various ways, the participants who are directly or indirectly affected by the global and local changes inherent in the transformation process.

This is a wider trawl of participants than the organisational stakeholders than commonly identified in "soft systems methodologies" for use by organisational analysts (e.g., Checkland and Scholes, 1990; Jackson, 1991) as it takes into account not only those considered to have a stake in the "transformation problem", as first identified, but also those who would experience the results of the actions taken in handling or "solving" the problem, and those involved in the results and side effects of the implementation of any "solution".

As a basis for communicative action (Habermas, 1990) all participants, thus defined, will, ideally, need to provide information about "what they know best". It is also necessary to organise, and feed back, on a distributed basis, an enhanced understanding of this collective knowledge, given that a suitable vehicle of communication could be found for this purpose. The major difficulty facing this enterprise is to elicit the knowledge participants possess in a way that understanding can be achieved, since disparate assumptions about possibly shared knowledge and interpretative frameworks (as commonly accepted within the organization) are likely to flavour individual participants' discourse. 
Hence any effective vehicle of communication must orient the discourse within an interpretative framework where each participant's assumptions about what is (or assumed to be) commonly known within the organization can surface (McCaskey, 1988). The choice of the appropriate interpretative framework is a matter of sensitivity to local cultural and social conditions within the lifeworld of the organization (Habermas, 1989). It is not appropriate to presume the hegemony of the interpretations and assumptions of any particular group or stratum, such as top management, in this respect.

\subsection{Sensitivity to local cultural and social conditions}

In searching for the appropriate interpretative framework in any particular organisational context, the "organisational culture metaphor" is often invoked: i.e., the common notion that "culture is a determinant of social life in any particular context and that, by extension, organisational culture determines the interpretative patterning of organisational life (Handy, 1985). Essentially, "culture", itself a metaphor taken from agriculture, is a construct, controversial at base, under which a lot of different things may be glibly placed, explaining mainly differences between various groups. Emphasis on differences has obscured the realisation, fundamental to the agricultural use of the term, that culture is a process (originally of cultivating the land).

Individual organisations, which would seem to be situated within the same general cultural context, have their own cultures with a great deal of subcultural variation. Morgan (1986) identifies the fundamental criterion for the existence of an organization, in functional, process terms, to be the enactment of $a$ shared reality. It is this enactment process which constitutes the "organisational culture" as a set of shared assumptions informing observation and action. It is maintained through formal and informal interactions between the participants in the organization processes and is expressed in human relations.

Nevertheless, organisations contain subcultures; thus enactment processes may be shared within particular groups or strata within an organization (c.f. Jaques, 1989), but not be homogeneous across them. Thus overall, organisational culture is patterned according to local conditions. Great differences may exist between different groups of people within the organization in terms of the expectations, assumptions, rules, etc. to which they ascribe. The resulting clashes and miscommunication can create problems for the organization as a whole. On the other hand, "cultural differences" may exist between groups of individuals which are external to the enactment process within the organisational context in which they work (e.g., age, sex, background, education, nationality, etc.). Although these differences can bring with them different expectations and assumptions, to the extent that these individuals can play their part in the enactment process, they may succeed in working together in an approximate equilibrium (Bateson, 1973).

Sensitivity to the existence of potential differences in organisational subcultures is often not sufficient if it is expressed only in terms of what can be observed and 
inferred: it must also address local enactment processes. Adopting this view means articulating the language of action as well as the language of observation in accessing this culture in enactment (de Zeeuw, 1993). The construction and maintenance of a shared reality allows this articulation to provide a vehicle of communication between participants who may yet have, and maintain, different perspectives and preferences during a successful process of organisational transformation.

\subsection{Implementability and local enactment processes}

According to our ideas, organisational transition (independently of the level in the organization at which this transition takes place) differs from mere organisational dynamics precisely through the need to design and use a new perspective, a new outlook (Humphreys and Nappelbaum, 1989, Humphreys and Berkeley, 1995). In respect of this outlook, some of the parameters of the organization activities that were previously largely irrelevant become crucially important and even central. In this sense, any movement within a pre-established state space corresponds to system dynamics, to quantitative, purely parametric changes while organisational transition is bound to involve changes of the state space itself which are not merely implicate changes in the transition function but rather presupposes some changes in the state variables themselves.

It has recently become fashionable when speaking about organisational transition and organisational change, to emphasise the role of implementability and of verifying implementability as one of the main prerequisites for a full-fledged systems analysis of the problem and its suggested solution.

In terms of the logic of problem formulation and choice (Nappelbaum, 1997) implementability, along with the higher principles of work design (Hackman and Oldman, 1980) is the main factor in reducing the versatility of problem perceptions to a sole choice to be realised in a subsequent course of actions. Curiously, however, in the analysis of organisational choice, both the implementability of the policy of organisational transition and the design of the problem perception itself remain on a purely personal basis. Indeed, in nearly every methodology has been proposed as suitable for importation to Eastern Europe to improve organisational transformation (e.g. organisational strategic planning and management systems, soft systems analysis methodology, decision conferencing, see section 2.0, above) only one overall problem formulation and hence only one systems perspective is sought for and the actions proposed to "solve" the problem are checked only for their horizontal implementability, i.e. for implementability within the context established through this perspective.

Meanwhile any organisational transition involves scores of individual and group perspectives to be developed and many individual and group problems to be formulated and acted upon in the process of individual and group adaptation to the new organisational policies and structures which have been adopted by top management, and to the new structures and styles that follow it. Implementation of 
organisational transformation is thus fundamentally located at the level of local enactment processes, and the resulting overall transformation is, for better or for worse, a synthesis of the outputs of these processes. Kieser (1997) identifies reasons why the authors of, and consultants on, such methodologies find this convenient for themselves.

\subsection{Some consequences}

The picture we have developed above of the preferred approach to organisational transformation in the emerging "Westernised" climate of Eastern Europe is not a happy one. Indeed it has engendered a very high failure rate in organisational transformation, resulting in many bankrupt organisations, and in some cases threatening to wipe out indigenous organisational activity in entire sectors. However, it is extremely essential, in this context, not to despair, not to consider that this prevalence of organisational failure should compromise the very idea of transformation and reform in Eastern Europe. Drawing such a conclusion, and acting accordingly, would be to undermine the trust between people - participants in organisations - that generated the hope and motivation for the overall transformation process in the first place.

If the general process of organisational, and societal, transformation is to build upon rather than subvert this trust, then, in our opinion a radical reconceptualisation of how this process is to be planned, analysed and implemented is required, quite different from the current "preferred approach" which we have reviewed above. In particular, as a result of this reconceptualisation:

- Participants in organisational processes are viewed as active change agents (valuable resources) rather than workforce (usable and disposable commodities). The focus is resource enhancement in cultural transformation, rather than resource utilisation in structural transformation.

- Discourse on organisational change will involve articulation of the language of action as well as the language of observation .

- Organisational personnel employed on productive tasks may also take up the role of the analyst, for a while: observing, communicating and acting with the aim of improving their own and the organization working conditions.

\subsection{IMPLICATIONS FOR ORGANISATIONAL PROCESS MODELLING AND DECISION SUPPORT}

It is not necessary that all assumptions be articulated concerning local enactment processes for a shared understanding, requisite in the context of organisational 
transformation, to be reached by participants in those processes. Neither is it possible, or desirable, to capture all relevant knowledge that exists within the organization. For successful organisational process model development, the fundamental issue is to discover, during moments of reflexivity, where one has reached a satisfactory understanding to be able to proceed further (on a conditional basis) with the analysis and synthesis of the material gained from organisational personnel that far during the process of the investigation.(Willis, 1980).

This (conditional) analysis should not aim to produce "complete" or "final" results. Rather, its aim is to increase the competence of the analyst to make accessible knowledge informing the actions of the participants in a way that it can be used as a creative resource by them in any synthesis. In order to come to understand the inherent processes, meanings and distortions, any methods used need to be flexible, sensitive and open, rather than prescriptive, at this level (Kersten, 1986).

\subsection{Investigation, analysis and creative synthesis}

The investigation process is typically considered the precursor of an analytic process whereby the organisational structure and procedures are investigated. However, an analytic activity which addresses all relevant aspects of an organization and which structures this information to represent "what is" in the realm of the participants' discourse still needs to be complemented by a synthetic activity: putting diverse parts of this information, which should be linked in reality, back together in various different configurations (checked for consistency and coherence) to find out "what could be" in actual, or potential (future) reality.

It is important that any actions and plans which emerge are based on investigation of the "real world" of the organization functioning (not on a world which simply structures participants' discourse). The basis for the preferred actions, the implementation route, and their potential side effects, need to be described and explored in a language to which stakeholders have access, and which extends their own discourse, thus offering them additional resources for communicative action (De Zeeuw, 1993, De Zeeuw and Schuurman, 1997).

This strengthens, in any local context, the possibility of taking informed action through Habermas' ideal process of "a forum of speakers exhibiting communicative competence (Jackson, 1991). It also obviates the need to reduce stakeholders from the role of active participants to that of merely "witnesses" (Ulrich, 1988) since the additional resources extend the language which they can use, within the context of the local conditions and culture to provide them, in practice, with the very competence which Ulrich (preferring to promote local cultural hegemony of the analyst's interpretation and language) rejected as "impractical", just as Eastern (and Western) European top management today often reject proposals for developing and exercising such competence within their organisations as "impractical". 


\subsection{Support for the analysis and synthesis of organisational systems}

Two major technological developments have made it possible for this idea to be implemented. The first one is the widespread dissemination of communications networking. The second must is a possibility of approximate modelling with variable degrees of precision in presentation, as well as, and independently from, traversing (for investigating horizontal implementability) and zooming and unzooming (for investigating vertical implementability). Few Decision Support Systems with this kind of capability have yet succeeded in practical application within implementation of organisational transformation according to this idea. One exception is SASOS (Support for the Analysis and Synthesis of Organisational Systems, c.f., Humphreys and Berkeley, 1992) and so, in the following, we indicate some key design concepts which provided SASOS with this capability.

\subsubsection{Views on organisational reality informing SASOS}

Organisational reality is a complex entity, synthesised through the language and perspectives and shared assumptions of those who experience that reality. This "reality" is constantly transforming itself as people interact, activities are carried out, and change is affected by the mere passage of time. Any participant in this "reality", taking up the role of analyst for the first time, or any external, professional analyst, inevitably discovers, if he or she takes the effort, that it takes a considerable amount of time to come to learn about this reality and the rules under which it functions and the elements which constitute it.

The learning process involves not only the conventional focus of organisational analysis, i.e., observing the way that organisational activities transform resources. It also involves being told stories about the processes and actions which, by their very nature, are not accessible through observation (de Zeeuw, 1993) or being told about it:.

This kind of information needs to be initially collected and exchanged in the form of commentaries coming from different people, with different responsibilities, perceptions, and experiences within the organization. Such organisational discourse may include elements which contradict each other (even if only by virtue of the fact that they have been voiced by different persons) which the analyst has to make sense of. The commentaries address problems arising, the activities involved, the results (intended or otherwise) and side effects, the characteristics of the resources involved, or required, and so on. They often appear fragmented, difficult to integrate at once into a conceptual model (Checkland, 1981), and yet have the potential to provide the seeds for important lessons to be learned.

SASOS i.e. employed in organisational analysis to address the complexity of the myriad of local enactment processes which collectively constitute an organization in transition by encouraging its users to take partial views on this reality according to their own local knowledge, desires and experience, to describe their characteristics relationships and linkages. It offers support for synthesising 
what has been described to enable the tracing of how problem situations may arise, and the development of actions to transcend them.

Taking a partial view is a functional way of bringing to the fore certain aspects of this reality and considering them in detail while paying less attention to other aspects of it which, although important in understanding it, fall within another domain of concern. SASOS is organised around three different views that can be taken on organisational reality, determined by the three different types of information an analyst requires to carry out his or her work (Berkeley, Humphreys and Quek, 1992). Each view presents a different, although not independent, picture of this reality, provides different types of information, and provides opportunities for different types of usage of this information.

Each view is guided by a different motivation, it addresses a different analytic goal or creative desire and, thus, the exit point from model development within that view can be easily decided upon by the user himself or herself on the basis of whether, in his or her opinion, the particular goal or desire has been achieved (even if only tentatively), or is still to be achieved. Thus, SASOS can be experienced as flexible and responsive to the current needs of the individual in this work as it collectively progresses, rather than just being seen as a prescriptive device.

The three views are:

- A static entity-relationship view, which links and structures information from participants concerning "observable "entities and their relationships which define the organization and its function within the general environment;

- A process view, which links and structures information on the processes and actions that are carried out within the organization and the various functions of the organization

- A systemic view, which provides information on comments voiced and stories told by participants about existing and potential problems in the organisational lifeworld and about desirable future situations and potential pathways which could improve current conditions.

While it may be convenient to start the process of organising the information provided a participant within a particular view, care should be taken not to subsume the analysis, as it develops, under just one view. Any single view, naturally, presents only a fragmented picture of the organisational reality orienting itself under one particular concern. Moreover, all three views are different ways of taking a partial look at the same organisational reality. It is only through the combined results of the material elicited by taking all three views, that one can, potentially, discover all the relevant aspects of the organization which are important in the analysis and creative synthesis of the structures and processes which will emerge from the organisational transformation. 


\subsubsection{Representation means sensitive to local cultural and social conditions}

SASOS's representation means provide a vehicle of communication for intuitive understandings about processes in the static entity-relationship view, where they can be only inferred intuitively, not observed. It enables them to be precised through employing the language of action (precising relations and rules of change) without needing to increase the formality of the (static) descriptions of the entities involved in these processes.

These descriptions remain in the form of the narrative text from accounts given by organisational participants, and articulates their own language. There is no need for an analyst to interpret this text according to his or her own (local culture and motivation bound) assumptions in translating the language of the participants into some kind of formal extension of his or her own observational language'. All that changes is how the narrative hypertext structure is cross-referenced to elements and regions of the process model as it is generated through the actions of an analyst interacting with SASOS.

While SASOS offers support for process model generation at all levels of precision from exploration of hypertext at one extreme to simulation of predicatetransition nets (Genrich, 1987) at the other, this is not the only kind of support which is requisite to improve the communicative competence of the analyst and the other organisational participants involved. It is also necessary to support the visualisation of what is being modelled, in a way that is sensitive both (i) to what the user's (viewer's) visualisation problem is, and (ii) to the kind of visualisation language which will promote an efficient and appropriate interpretation of what is "seen" through its use. The latter, of course, will be highly dependent on the local culture, the social conditions determining the use of the "views" thus provided, and the (un)familiarity of the participants with representation techniques which employ particular kinds of visualisation language.

Hence, SASOS provides for the particular visualisation language and techniques employed in any context when working on, or viewing a process model both (i) tc match the user's visualisation problem and (ii) to be customised to employ a visualisation language which appears "natural" to the participants who view what SASOS can show them. It is these customisation facilities, offered by SASOS, which ensure its ability to support and guide the user in a modelling enterprise that will effectively increase participants' communicative competence in discourse on organisational transformation.

\footnotetext{
'Thus, within the scheme described by de Zeeuw, 1993, this enables the synthesis of extensions to participants' own language(s), l.'s, rather than utilizing some extended language $L$ ' which may work against any such synthesis.
} 


\subsubsection{Zooming and unzooming within variable precision modelling}

As well as variable precision modelling, SASOS's representation means offer zooming (refinement) and unzooming (coarsening) facilities over the whole range of information to which it has access, regardless of the level of precision at which this information is represented (from hypertext to exact nets). ${ }^{2}$ The need for these facilities is more or less obvious. Participants in different levels of the organisational structure use different, though "nested" contexts to develop their perceptions of the small world they have to operate within ${ }^{3}$, and of the local enactment processes within that world.

Exercise in the concurrent development of the entire set of these perceptions supported by SASOS' machinery of variable precision modelling and zooming and unzooming facilities can be instrumental in:

- facilitating the development of local perceptions on different levels of organisational structure and hence checking the vertical implementability of any policy developed at the top, and contributing, bottom-up, to its construction and refinement;

- helping lower levels of the organisational structures to dispel their fears and phobias about impeding changes and in shortening the adaptation period;

- unclogging the middle management bottleneck mentioned in section 2.2, above;

- opening the possibility of top level policy adjustment that will allow for the special interests of lower level stakeholders and actors and hence removing unnecessary barriers on the implementation path for the top level policy.

${ }^{2}$ See Richter et.al., 1987 for a detailed discussion, with examples, of the distinction refinement and precision in organizational conceptual model development and exploration.

'The idea of "nested perceptions", however, is by no means clear. The rules that govern nestedness or internal compatibility of differing scale perceptions is not only central in providing the kind of support but also for the whole concept of cognitive closure. It is clear that approximation mentioned above in this case does not amount solely to shedding certain details or to disaggregation of certain aggregated variables used in a higher level perception. In supplementing these with some other components (that may be totally foreign to the higher level perceptions) it creates its own concepts in which "borrowed" components may be differently interpreted and evaluated. 
There are at least two distinct cases of incompatibility that may hamper vertical implementability of the transition policy referred to earlier:

- it may involve opposite value judgements of the same cognitive component interpreted differently on different levels; or

- it may involve emergence of a neglected feature in a higher level perspective as a result of aggregation of representations developed below.

It is essential also to understand that the vertical compatibility mentioned above does not possess any monotonicity or transitivity in a sense that some lower level perception that is perfectly compatible with its immediate predecessor may prove to be incompatible with some higher perception even if it is fully compatible with its immediate predecessor ${ }^{4}$

At the same time, within the organisational framework, these issues deserve special attention and should allow also for the specific social climate of a particular organization, its scale, its built-in culture and cultural differences between various levels and communication problems that exist and can be both alleviated and aggravated by the emergence of the instrument of a kind we have just suggested. Decision support systems that would develop (among others) the capabilities we have been trying to focus upon may deserve the name of organization transition support systems.

\section{CONCLUSION}

In this paper we have traced the high failure rate of attempts at organisational transformation in Eastern Europe, in part at least, to the desire of top management to import, and employ, techniques for analysing the conditions of their organization and formulating prescriptions for change in which subjectivity, while recognised, is divorced from organisational setting. This serves to reinforce the domestication of the productive participants in their organization and to undervalue to the knowledge they possess about local enactment processes, and how they might be improved.

We have proposed an alternative approach in which the process of creative transformation of organisations can be brought to centre field, and have shown how

\footnotetext{
${ }^{4}$.The issue of vertical compatibility and implementability outlined earlier may appear to relate only to decision making of strategic purport within an organisational framework. However, we believe that it is equally important for purely personal decisions, because thinking about implementation of personal decisions also involves focusing and defocusing, framing and reframing and zooming and unzooming.
} 
support may be provided to organisational personnel at all levels (not just top management) in this process of creative transformation, promoting, rather than restricting, analysts' and management's abilities to be sensitive to local cultural and social conditions.

We have explored the implications of this understanding for organisational process modelling, starting from the need to discover, not to assume, what to observe and to articulate the language of action as well as the language of observation in accessing the culture in enactment. The aim is to support not only organisational analysis but also creative synthesis

of possibilities for organisational change and transformation. We believe that the need to defocus on this aim is an urgent priority in order to arrest and reverse the general process of organisational decline which we are currently witnessing not only in Eastern Europe, but also in many other parts of the industrialised world.

\section{REFERENCES}

Bannon, L. (1997) CSCW - A challenge to certain (G)DSS perspectives on the role of decisions, information, and technology in organizations? This volume.

Bateson, G. (1973) Steps to an ecology of mind. Paladin, St. Albans:.

Beisinger, M. R. (1988) Scientific management, socialist discipline and Soviet power. Tauris, London:

Berkeley, D. de Hoog, R. and Humphreys, P.C. (1990) Software development project management: process and support. Ellis Horwood/Simon and Schuster, Chichester.

Berkeley, D. Humphreys, P.C. and Quek, F. (1992) Dynamic process modelling for organisational systems supported by SASOS, in Dynmod 3: proceedings of the third international conference on dynamic modelling of information systems. ( eds. H. G. Sol and A. Verbraek ), Technical University Press, Delft.

Chandler, A. D., Jr. (1969) Strategy and Structure. Cambridge, MIT Press, Cambridge, Mass.

Checkland, P. (1981) Systems Thinking, Systems Practice. Wiley, Chichester.

Checkland, P. (1994) Conventional wisdom and conventional ignorance: the revolution organization theory missed. Organization, 1, 29-34.

Checkland, P. and Scholes, J. (1990) Soft systems methodology in action. Wiley, Chichester.

DeSanctis, G. and Dickson, G. W. (1987) GDSS software as "shell" system in support of a programme of research, in Proceedings of 20th annual Hawaii conference on systems sciences.

Cooper, R. and Burrell, G. (1988) Modernism, postmodernism and organizational analysis: an introduction. Organization Studies, 9, 91-112.

Espejo, R., 1989. A method to study organisations, in The viable system model. (eds. R. Espejo and R. Harnden ) Wiley, Chichester. 
Fekete-Szucs, L. (1991) Decision conference for strategic issues: theory in practice, in Environments for supporting decision processes (eds. H. G. Sol and J. Vecsenyi) Elsevier, Amsterdam.

Genrich, H. J. (1987) Predicate/Transition nets, in Petri Nets: Central Models and their properties (eds. W. Brauer, W. Reisig and G. Rozenberg) SpringerVerlag, Berlin.

Habermas, J. (1989) The Tasks of a Critical Theory of Society in Critical theory and Society: a reader (eds. S. E. Bronner \& D. M. Kellner) Routledge, London.

Habermas, J. (1990) The theory of communicative action. Volume 1: Reason and the rationalisation of society. Polity press, London.

Hackman, J. R. and Oldham, G. R. (1980) Work redesign. Addison Wesley Reading, Mass.

Handy, C. (1985). Understanding organisations. Penguin, London. (3rd ed.)

Humphreys, P.C. (1984) Levels of representation in structuring decision problems. Journal of applied systems analysis, 11, 3-22.

Humphreys, P. C. (1989) Intelligence in decision support: a process model, in Knowledge based management support systems (eds. G. Doukidis, F. Land and G. Miller) Wiley, Chichester.

Humphreys, P.C. and Berkeley, D. (1992) Support for the synthesis and analysis of organisational systems in deciding on change, in Decision support systems: Experiences and expectations (eds. T. Jelassi and W. Mayon-White) Elsevier, Amsterdam.

Humphreys, P.C. and Berkeley, D. (1986). Organisational knowledge for supporting decisions, in Decision support systems: a decade in perspective (eds. E. R. McLean and H. G. Sol) North Holland, Amsterdam.

Humphreys, P.C. and Berkeley, D. (1995) Organisational process modelling sensitive to local cultural and social conditions, in Work and organizational psychology: European contributions of the nineties (eds. J. M. Piero, F. Prieto, J. L. Melia and O. Luque) Erlbaum (UK) Taylor and Francis, Hove.

Humphreys, P.C., Berkeley, D. and Jovchelovitch, S. (1996) Organisational psychology and psychologists in organisations: Focus on organisational transformation. Interamerican journal of psychology, 30, 27-42.

Humphreys, P.C. and Nappelbaum, E. L. (1989) Strategic Analysis of organisations in transition: knowledge structuring, conceptual modelling and implementation of changes. Organisational Research Group, LSE, London.

Jackson, M. C. (1991) Systems methodology for the Management Sciences. Plenum, New York.

Jaques, E. (1976) A general theory of Bureaucracy. Heinemann, London.

Jaques, E. (1989) Requisite organization: the CEO's guide. Casson Hall, Arlington, Virginia,

Keen, P. G. W. (1986) Decision support systems: the next decade, in Decision support systems: a decade in perspective (eds. E. R. McLean and H. G. Sol) North Holland, Amsterdam. 
Kersten, A. (1986) A critical-interpretative approach to the study of organizational communication: bringing communication back into the field, Organization $<>$ Communication: Emerging perspectives in (ed. L. Thayer) Ablex Norwood, New York.

Kieser, A. (1997) Rhetoric and myth in management fashion. Organization, 4, 49-74.

Lederer A. L., and Sethi, V. (1988) The implementation of strategic information system planning methodologies. MIS Quarterly, 12, 445-461.

McCaskey, M. B. (1988) The challenge of managing ambiguity and change, in Managing ambiguity and change, (eds. L. R. Pondy, R. L. Boland, Jr and H. Thomas) Wiley, Chichester.

Masaaki, I. (1989) Kaizen: the key to Japanese competitive success. Random House, New York.

Maturana, H. and Varela, F. (1979) Autopoesis and Cognition. Reidel, Boston.

Merkle, J. A. (1980) Management and ideology: the legacy of the international scientific management movement. University of California Press, Berkeley:

Mitroff, I. I. (1983) Stakeholders of the organisational mind. Jossey-Bass, San Francisco.

Morgan, G. (1986) Images of Organization. Sage, London.

Mumford, E. (1991) Decision making and the organisational environment: today's problems and tomorrow's needs, in Environments for supporting decision processes (eds. H. G. Sol and J. Vecsenyi) Elsevier, Amsterdam.

Nappelbaum, E. (1997) Systems logic for problem formulation and choice. This volume.

Nelson, D. (1980) Frederick W. Taylor and the rise of scientific management. University of Wisconsin Press, Madison.

Paprika, Z. and Zoltay, A. (1994) The Current Hungarian Business Climate. BEAMS Doc. UR-RR-006, Department of Business Economics, Budapest University of Economic Sciences, Budapest.

Phillips, L. D. (1989) People-centred group decision support, in Knowledge based management support systems (eds. G. Doukidis, F. Land and G. Miller) Wiley, Chichester.

Phillips, L. D., 1992. Gaining corporate commitment to change, in Executive information systems and decision support (ed. C. Holtham) Chapman and Hall, London.

Richter, G., Humphreys, P.C., Voss, K., Berkeley, D., Genrich, H., Domke, M., Griebler, H. and Wisudha, A. (1987) GOFOR: Generic Office Frame of Reference. Annex to Final report, ESPRIT project 56. CEC, Brussels. (Abbreviated version published as Chapter 4 in G. Schafer (ed),op. cit)

Rose, N. (1989) Governing the soul: The shaping of the private self. Routledge, London:

Schafer, G. (1988). Functional Analysis of Office Requirements: A multiperspective approach. Wiley, Chichester. 
Taylor, F. W. (1947) Scientific Management. Harper and Row, New York.

Ulrich, W. (1988) Systems thinking, systems practice and practical philosophy: A programme of research. Systems Practice, 1, 137-163.

Vari, A., Rohrbaugh, J. and Baaklini, A. I. (1992) Decision conferencing GDSS in the Hungarian Parliament: A case of systems planning, In Decision Support systems: Experiences and expectations (eds. T. Jelassi, M. R. Klein and W. M. Mayon-White ) Elsevier, Amsterdam.

Vari, A, and Vecsenyi, J. (1984) Selecting decision support methods in organisations. Journal of applied systems analysis, 11, 25-36.

Willis, P. (1980) Notes on method, in Culture, media, language (eds. Hall, S., D. Hobson, A. Lowe and P. Willis) Hutchinson, London.

Wrege, C. D. and Greenwood, R. G. (1991) Frederick W. Taylor, the father of scientific management: Myth and reality. University of Illinois press, Homewood, Illinois.

Yetton, P. and Crawford, M. (1992) Reassessment of participative decision making: a case of too much participation, in Decision making and Leadership (ed. F. Heller) Cambridge university press, Cambridge.

de Zeeuw, G. (1993) Soft knowledge accumulation and the rise of competence. Systems Practice, 5, 192-215.

de Zeeuw, G. and Schuurman, J. G. (1997) Support as intervention in decision processes. This volume.

\section{BIOGRAPHY}

Patrick Humphreys is a founding member of IFIP working group 8.3. He is Professor of Social Psychology at the London School of Economics and Political Science, where he convenes graduate courses on Organisational Social Psychology, Decision Making and Decision Support Systems and the Social Psychology of the Media. He led the LSE teams on CEC ESPRIT projects on Functional Analysis of Organisational Requirements and on Project Integrated Management Systems and directed the CEC TEMPUS project BEAMS - Business Economics and Management Support and many other projects on decision making, organisational process modelling, networking and communication support. His books include How Voters Decide, Analysing and Aiding Decision Processes, Effective Decision Support Systems, Exploring Human Decision Making and Software Development Project Management: Process and Support.

Eric L. Nappelbaum is Vice Chancellor at the Moscow School for Business of Political Science and Law and Research Director at the Moscow Institute of System Analysis, faculty of Operational Research. He studied Physics and Computer Sciences at the Moscow State University and did his PhD on Control 
Problems at the Moscow Physical Technical Institute. Dr Eric L. Nappelbaum is member of All Russian Academy of Science , Department of Operational Research and IT Strategy. Dr Eric Nappelbaum has published more than 40 international papers. He is Visiting Professor at the London School of Economics and Political Science and at the International Management Centre in Budapest. The research interests of Dr Nappelbaum include Organisational Transformation, System Analysis and System Logic. 